\title{
Application of Mid-infrared Spectroscopy for the Measurement of Several Quality Parameters of Alcoholic Beverages, Wine and Raki
}

\author{
Burcu Ozturk • Dila Yucesoy • Banu Ozen
}

Received: 23 December 2011 / Accepted: 7 March 2012 /Published online: 23 March 2012

(C) Springer Science+Business Media, LLC 2012

\begin{abstract}
Mid-infrared (IR) spectroscopy, which is a rapid and relatively small amount of waste producing technique, was used to predict several quality parameters of two types of alcoholic beverages, wine and raki. Mid-infrared spectra of red, rose and white wines and a traditional aniseed alcoholic beverage, raki were collected and relations were established between measured chemical parameters $(\mathrm{pH}$, brix, total phenol content, anthocyanin content, titratable acidity, sugar content, electrical conductivity and some colour parameters) of these beverages and their infrared spectra using chemometric techniques. Partial least square regression provided excellent prediction of total phenol $\left(R^{2}=0.97\right)$ and anthocyanin contents $\left(R^{2}=0.98\right)$ of wine samples and a good prediction of $\mathrm{pH}\left(R^{2}=0.9\right)$, brix $\left(R^{2}=0.92\right)$ and colour intensity $\left(R^{2}=0.93\right)$ values were obtained. Brix, total phenol and sugar content of raki samples were also estimated very successfully $\left(R^{2}=0.99\right)$ for raki and good prediction was obtained with $\mathrm{pH}$ value. Mid-IR spectroscopy in combination with chemometrics could be a promising technique for determination of several quality parameters of alcoholic beverages simultaneously and rapidly.
\end{abstract}

Keywords Infrared spectroscopy $\cdot$ Chemometrics · Partial least squares $\cdot$ Wine $\cdot$ Raki

\section{Introduction}

Wet chemical methods for qualitative or quantitative determination of different chemical compounds or indices,

B. Ozturk $\cdot$ D. Yucesoy $\cdot$ B. Ozen $(\bowtie)$

Department of Food Engineering, Izmir Institute of Technology,

Urla-Izmir, Turkey

e-mail: banuozen@iyte.edu.tr require time consuming, waste generating, laborious and expensive procedures (Bevin et al. 2006). That's why there is a need of simple and rapid methods that can be easily applied in routine laboratory analysis. With the advantages of short analysis time and high resolution, there are several examples of the use of mid-infrared (mid-IR) spectroscopy for both quantification and classification of wines and other alcoholic beverages in literature (Moreira and Santos 2004; Pontes et al. 2006; Lachenmier 2007).

Mid-IR spectroscopy can be used in the differentiation analysis since the IR spectrum of a compound is a characteristic property which can be accepted as a 'fingerprint' (Ismail et al. 1997). Therefore, Mid-IR spectroscopy has applications in the classification of several food products. For instance, this technique was successfully utilised in differentiation of the 'Fino' sherry wines according to six different ageing levels (Palma and Barroso 2002). Quantitative analysis can be also applied with the use of mid-IR spectroscopy technique on the account of the fact that the amount of the IR energy absorbed by the individual component is directly proportional to the amount of each compound present (Ismail et al. 1997). Mid-IR spectroscopy was applied to fermented Chenin Blanc and synthetic musts for the quantification of volatile acidity, glycerol, ethanol, reducing sugar and glucose concentrations (Nieuwoudt et al. 2006). Excellent predictive accuracy was achieved for the prediction of volatile acidity and regression of ethanol, reducing sugar and glucose ended up with a satisfactory prediction model (Nieuwoudt et al. 2006). In another study, this spectroscopic technique combined with chemometric methods was applied for the quantitative analysis of red wine tannins and the investigation resulted in accurate predictions (Fernández and Agosin 2007). Some quality parameters of beer such as density, ethanol content, $\mathrm{pH}$ and bitterness unit were also tried to be predicted from mid-IR 
spectra (Lachenmier 2007). Strong correlations were established between IR measurements and density, ethanol, methanol, ethyl acetate, propanol-1, isobutanol and 2-/3-methyl1-butanol.

The objective of this study is to investigate the potential use of mid-IR spectroscopy in combination with chemometric techniques for the prediction of certain quality parameters of a fermented and a traditional distilled alcoholic beverage.

Wine was chosen as the fermented beverage while raki, a traditional aniseed beverage from Turkey was the distilled beverage. Raki is a spirit that is produced by double distillation of suma or suma mixed with agricultural-based ethanol and flavoured with aniseed (Pimpinella anisum). Suma is produced mainly from raisins, molasses and/or grape must and is a distillate with a maximum $94.5 \%$ ethanol content. Suma can be also mixed with ethanol from an agricultural origin and this final mixture must contain at least $65 \%$ suma. Suma or suma-ethanol mixture is distilled in copper alembics with a maximum capacity of 5,000 1 and three groups of distillates, head, medium and tail, are separated (Anli and Bayram 2010). Medium distillate is used for raki manufacture and diluted to $45 \%$ alcohol. After the addition of sugar product is stored for at least 1 month before filling.

\section{Materials and Methods}

\section{Samples}

A total of 79 samples from mono-varietal Turkish wines belonging to 13 different varieties were used in the study. To cover a wide range of variety, dry and semi-sweet wines produced from the grapes of all winery regions in Turkey were selected. Forty-six red (Bogazkere, Cabernet Sauvignon, Kalecik karasi, Merlot, Okuzgozu, Papazkarasi and Shiraz varieties), 28 white (Emir, Muscat, Narince, Sultaniye and Chardonnay varieties) and five rose (Calkarasi and Okuzgozu varieties) wines belonging to 2006 and 2007 vintages were bought from the local markets in Turkey. The wines were stored at $4{ }^{\circ} \mathrm{C}$ throughout the study.

Raki samples were also obtained from local markets and cover most of the brand names produced in Turkey. Two ouzos, an aniseed drink from Greece, were also included into the sample set. Totally 34 distilled beverages were analysed. The chemicals used in the experiments were all analytical grade.

\section{Mid-IR Spectroscopic Analysis}

All samples were scanned through a Fourier-transform infrared (FT-IR) spectrometer (Perkin Elmer Spectrum 100 FT-IR spectrometer, Wellesley, MA) with a DTGS detector within the range of 4,000-650 $\mathrm{cm}^{-1}$. Measurements were performed with a horizontal attenuated total reflectance attachment (HATR ZnSe 45 deg. Trough Plate) of the instrument. The scanning was carried out at $4 \mathrm{~cm}^{-1}$ resolution and at $0.50 \mathrm{~cm} / \mathrm{s}$ scan speed. The number of scans for each spectrum was adjusted to 64 . During scanning the sample holder was covered with a lid to prevent evaporation of the alcohol. The sampling crystal was cleaned with ethanol and distilled water after each measurement and dried under nitrogen flow. The measurements were repeated at least three times.

\section{Reference Analysis}

\section{Total Phenol Determination}

Total phenol contents of the wine and raki samples were determined with the use of Folin-Ciocalteau micro method, a method derived from total phenol analysis (Slinkard and Singleton 1977). Forty microlitres of white wine or raki and $3.16 \mathrm{~mL}$ of distilled water was mixed, then $200 \mu \mathrm{L}$ of FolinCiocalteau reagent was added into this mixture. After $4 \mathrm{~min}$, $600 \mu \mathrm{L}$ of sodium carbonate $(0.2 \mathrm{~g} / \mathrm{mL})$ solution was also added and mixed. The solutions were kept for $2 \mathrm{~h}$ in a dark place at room temperature then the absorbance of each solution was read against the blank at $765 \mathrm{~nm}$ with a UVvisible spectrophotometer (Shimadzu UV-2450, Japan). The red and rose wines were firstly diluted by a factor of 10 to see the colour changes clearly, and then the same procedure was applied. Through the use of gallic acid calibration curve total phenol concentration of wines were calculated as milligrams of gallic acid per litre. The measurements were repeated three times.

\section{Anthocyanin Determination}

The total anthocyanin contents of wines were measured using AOAC $2005.02 \mathrm{pH}$ differential method. The anthocyanin content of the wines was determined by measuring the absorbances of the samples diluted with both $\mathrm{pH} 1.0$ and $\mathrm{pH} 4.5$ buffers at 520 and $700 \mathrm{~nm}$ with a UV-visible spectrophotometer (Shimadzu UV-2450, Japan). The anthocyanin concentration was expressed as cyanidin-3glucoside equivalents and it was calculated with the following formula:

$$
\begin{gathered}
\text { anthocyanin concentration }\left(\frac{\mathrm{mg}}{\mathrm{L}}\right)=\frac{A \times \mathrm{MW} \times \mathrm{DF} \times 10^{3}}{\varepsilon \times 1} \\
A=\left(A_{520}-A_{700}\right)_{\mathrm{pH}=1}-\left(A_{520}-A_{700}\right)_{\mathrm{pH}=4.5}
\end{gathered}
$$

where MW (molecular weight $)=449.2 \mathrm{~g} / \mathrm{mol}$ for cyaniding -3 glucoside, $\mathrm{DF}=$ proper dilution factor, $1=$ path length, $\varepsilon=$ molar extinction coefficient in moles per mole per centimetre for cyd-3-glucoside $(26,900), 10^{3}=$ factor for conversion from grams to milligrams. The measurements were repeated three times. 
Sugar Analysis

Sugar content of raki was measured using an HPLC (Perkin Elmer Series 200, Waltham, MA, USA) with a refractive index detector. Twenty microlitres of each raki sample were filtered through $0.45-\mu \mathrm{m}$ cellulose-acetate membrane filter (Sartorius, Germany) then injected directly into the HPLC; $0.045 \mathrm{~N}$ sulphuric acid containing $6 \%$ acetonitrile was used as mobile phase, with a flow rate of $0.5 \mathrm{~mL} / \mathrm{min}$. The column (Aminex, HPX-87C, $300 \times 7.8 \mathrm{~mm}$, Bio-rad) was maintained at $45{ }^{\circ} \mathrm{C}$. The identification of sugars was confirmed by using integrated peak areas according to external standard solution calibration. This method was adapted from Castellari et al. (2000).

\section{pH, Brix and Total Titratable Acidity Measurements}

$\mathrm{pH}$ and brix of the wine and raki samples were measured with a $\mathrm{pH}$ metre (HI221 Microprocessor $\mathrm{pH}$ metre, Italy) and a digital refractometer (Mettler Toledo Re50, USA), respectively. The titratable acidity measurements of the samples were performed according to AOAC 962.12 method. Titratable acidity of wines is expressed in equivalent of tartaric acid content (grams per litre). Measurements were repeated three times.

\section{Electrical Conductivity}

Electrical conductivity or specific conductance of raki was measured with a Bench Conductivity Metre (EC-215R Hanna Instrument, Italy). Before measurements conductivity metre was calibrated using its own calibration solution by adjusting the temperature coefficient from $0 \%$ to $2.5 \% /{ }^{\circ} \mathrm{C}$ (199.9 $\mu$ s ranges). Measurements were performed three times.

\section{Colour Measurements}

A spectrophotometric method (Kelebek et al. 2007) was utilised to measure the colour of the wine samples. Absorbance values at 420, 520, and $620 \mathrm{~nm}$ obtained with a UV-visible spectrophotometer (Shimadzu, Kyoto, Japan) were used in calculation of colour parameters. Several colour indexes were calculated using the following formulas:

Colour intensity (CI): $\mathrm{Abs}_{420}+\mathrm{Abs}_{520}+\mathrm{Abs}_{620}$

Tint: $\mathrm{Abs}_{420} / \mathrm{Abs}_{520}$

Proportion of yellow colour $-\mathrm{Ye}(\%)=\mathrm{Abs}_{420} / \mathrm{CI}$

Proportion of red colour $-\operatorname{Red}(\%)=\mathrm{Abs}_{520} / \mathrm{CI}$

Proportion of blue colour $-\mathrm{Bl}(\%)=\mathrm{Abs}_{620} / \mathrm{CI}$

Measurements were repeated three times.
Multivariate Analysis

The data from the FT-IR spectrometer was analysed using multivariate statistical techniques with SIMCA software (SIMCA P-10.5 Umetrics Inc., Sweden). Multivariate regression analysis was used to develop models to predict a property of interest. There are many examples of the use of modelling and prediction with regression analysis such as partial least square (PLS) method. PLS regression analysis can be used for relating two data matrices, $X$ (FT-IR spectral data) and $Y$ (analytical results of chemical parameters) by linear multivariate model (Eriksson et al. 2000). The main purpose of PLS regression in this study was to construct linear calibration models that enable prediction of chemical parameters like total phenol content, anthocyanin content, brix, titratable acidity, $\mathrm{pH}$ and certain colour parameters of alcoholic beverage samples using FT-IR spectral data.

The observation set was divided into calibration (2/3 of samples) and validation (1/3 of samples) sets. The calibration and validation sets were created with randomly selected observations and the results of the regression analysis were also visualised with prediction plots showing the regression correlation coefficient $\left(R^{2}\right)$ of the created models. The regression coefficient expresses the connection between predictions and the actual results of the chemical parameters and gives an idea about the predictive efficiency of the model (Bauer et al. 2008). The evaluation of the calibration models were performed by computing root mean square error of calibration and prediction (RMSEC, RMSEP) and residual predictive deviation (RPD). RMSEP is a measurement of the average difference between the predicted and reference actual values at the validation step. Similarly RMSEC describes predictive ability of calibration model with reference to the actual data. The RPD value is a significant criterion to be utilised for evaluation of the predictive ability of regression models (Esbensen et al. 2002). The calculations were performed by using the following formulas:

$\mathrm{RMSEC}=\sqrt{\frac{\sum_{i=1}^{n}\left(\hat{y}_{i}-y_{i}\right)^{2}}{n-2}}$

$\operatorname{RMSEP}=\sqrt{\frac{\sum_{i=1}^{n}\left(\hat{y}_{i}-y_{i}\right)^{2}}{n-1}}$

$\mathrm{RPD}=\frac{\mathrm{SD}}{\mathrm{RMSEP}}$

$\mathrm{SD}=\sqrt{\frac{\sum_{i=1}^{n}\left(y_{i}-y\right)^{2}}{n-1}}$ 
where $y_{i}$ is the actual value obtained from analytical methods for the $i$ th sample; $\hat{y}_{i}$ is the predicted value by mid-IR spectra for the same sample; $y$ is the mean of each set; $n$ is the number samples used in each set; SD is standard deviation in each set (Saeys et al. 2005; Zornoza et al. 2008). Accuracy of the prediction models were evaluated by using $R^{2}$, RPD and slope of the equations determined for the calibration sets (Table 1). In addition to these criteria; to construct a good model, the absolute values of RMSEC and RMSEP and differences between them should be small.

\section{Results and Discussions}

Mid-infrared spectra of wine and raki samples are provided in Fig. 1. FT-IR spectra show absorption bands at different frequencies and these bands are attributed to the various functional groups. The two distinct absorption bands at $3,600-3,200$ and $1,700-1,565 \mathrm{~cm}^{-1}$ are associated with $\mathrm{O}_{-}$ $\mathrm{H}$ stretching vibrations. The peak around $1,636 \mathrm{~cm}^{-1}$ corresponds $\mathrm{C}=\mathrm{C}$ stretch. The peak at $3,040-2,800 \mathrm{~cm}^{-1}$ is due to the stretching vibration of $\mathrm{C}-\mathrm{H}$ bond. The peaks between 1,565 and $965 \mathrm{~cm}^{-1}$ are in the fingerprint area where many different IR bands, including those corresponding to the vibrations of the $\mathrm{C}-\mathrm{O}, \mathrm{C}-\mathrm{C}, \mathrm{CH}_{2}, \mathrm{CH}_{3}, \mathrm{C}-\mathrm{OH}, \mathrm{C}-\mathrm{H}$ and $\mathrm{C} \equiv \mathrm{N}$ bonds occur (Lachenmier 2007; Ismail et al. 1997; Fernández and Agosin 2007). IR spectral peaks are related to the bonds in the compounds, therefore, could be correlated with the composition of a specific food sample such as its phenol, alcohol, aldehyde, higher alcohol, polyol, acid, sugar, volatile acid and amino acid content (Lee et al. 2009).

Wine and raki samples were divided into two groups, namely calibration and validation sets for the statistical analysis; $2 / 3$ of the samples were randomly selected for calibration models and $1 / 3$ for validation models. As a pretreatment step various spectral filtering techniques such as first derivation, second derivation and wavelet compression

Table 1 Criteria for the accuracy of prediction models (Tamaki and Mazza 2011)

\begin{tabular}{ll}
\hline Parameter & Criteria \\
\hline$R^{2}$ & Between 0.66 and 0.80 approximate predictions \\
& Between 0.81 and 0.90 good predictions \\
& $>0.90$ excellent predictions \\
& $<2.0$ insufficient \\
RPD & Between 2.0 and 2.5 approximate predictions \\
& Between 2.5 and 3.0 good predictions \\
& $>3.0$ excellent prediction \\
& $<0.8$ or $>1.2$ less reliable \\
Slope & Around $0.8-1.2$ reliable \\
& Between 0.9 and 1.1 very reliable \\
\hline
\end{tabular}

were applied to the raw data and the best results were obtained with orthogonal signal correction in combination with wavelet method. In addition, whole spectral range produced better results compared to selected wavenumber ranges. Selected spectral regions were $1,565-965 \mathrm{~cm}^{-1}$, $1,900-1,700 \mathrm{~cm}^{-1}$ and $3,040-2,800 \mathrm{~cm}^{-1}$ (Lachenmier 2007; Versari et al. 2010).

\section{Prediction of Wine Quality Parameters}

The chemical results including total phenol content, anthocyanin content, brix value, titratable acidity and colour values were provided as separate responses ( $\mathrm{Y}$ matrices) and models were developed for each of these responses separately instead of using all responses in a single model. The results of only red and rose wines were handled in anthocyanin regression analysis since anthocyanin only exists in red grapes. As the acidity terms are highly related to each other, the titratable acidity and $\mathrm{pH}$ results were analysed together. Similarly colour results were included to the regression analysis as one group. RMSEC, RMSEP, $\mathrm{RPD}$, slope and $R^{2}$ for all the PLS analysis for wine samples were calculated and summarised in Table 2.

PLS analysis for prediction of total phenol concentration was performed by relating FT-IR spectral data as X variables and total phenol content obtained with analytical methods as $Y$ variable. The model contains three significant components (PCs) explaining $96.6 \%$ of the total variation of total phenol content $(Y)$. The regression coefficient of the model determined with calibration set was found as 0.97 (Fig. 2a and Table 2). RMSEP value was also calculated with the validation set as 312.43 . According to comparison criteria set in Table 1, the determination of total phenol content by FT-IR yielded excellent prediction with slope of 0.97 , which accounts for high reliability, regression coefficient (0.97) greater than 0.9 and RPD value (4.08) greater than 3.0 (Table 2). In another study, red wines were analysed to determine the concentration of phenolic compounds during wine fermentation from the near-IR spectral data in combination with PLS regression analysis (Cozzolino et al. 2004). Cabernet Sauvignon and Shiraz wines from 2001 to 2002 vintages were analysed via HPLC as a reference method. The models provided good predictions of phenol concentrations using near-IR spectroscopy.

Prediction of anthocyanin content from FT-IR spectral data with PLS analysis was carried out with calibration and validation sets consisting of 32 and 16 observations, respectively. The regression analysis resulted in high correlation of $R^{2} 0.98$ for calibration set (Fig. 2b). The model includes three PCs explaining $97.5 \%$ of total variation $(Y)$ with a predictive ability of $91.5 \%$. The slope and $R^{2}$ value of the anthocyanin prediction model are 0.97 and 0.98 , respectively and they can be regarded as excellent and very 
Fig. 1 Mid-infrared spectra of $\mathbf{a}$ wine and $\mathbf{b}$ raki samples


reliable prediction. RPD value was calculated as 3.05, again resulting in an excellent prediction (Tables 1 and 2). Similar results were obtained in a previous study which was performed with 390 young red wines from Spain (Soriano et al.
Table 2 Summary of statistical results for PLS analysis of wine samples

\begin{tabular}{lccccrrr}
\hline Parameter & Number of PCs & $R^{2}(\mathrm{cal})$ & $R^{2}(\mathrm{val})$ & Slope & RMSEC & RMSEP & RPD \\
\hline Total phenol (mg/L) & 3 & 0.97 & 0.95 & 0.97 & 208.08 & 312.43 & 4.1 \\
Anthocyanin (mg/L) & 3 & 0.98 & 0.90 & 0.97 & 5.57 & 8.39 & 3.1 \\
Brix (\%) & 2 & 0.90 & 0.88 & 0.91 & 0.32 & 0.39 & 2.7 \\
Titratable acidity (g/L) & 5 & 0.89 & 0.73 & 0.89 & 0.22 & 0.32 & 1.7 \\
pH & 5 & 0.92 & 0.89 & 0.92 & 0.07 & 0.09 & 2.8 \\
Colour intensity & 3 & 0.93 & 0.92 & 0.93 & 1.12 & 1.63 & 2.8 \\
Tint & 3 & 0.71 & 0.54 & 0.71 & 1.03 & 1.41 & 1.4 \\
Yellow\% & 3 & 0.85 & 0.73 & 0.85 & 0.07 & 0.10 & 1.8 \\
Red\% & 3 & 0.83 & 0.67 & 0.83 & 0.06 & 0.09 & 1.7 \\
Blue\% & 3 & 0.73 & 0.84 & 0.73 & 0.02 & 0.02 & 2.2 \\
\hline
\end{tabular}



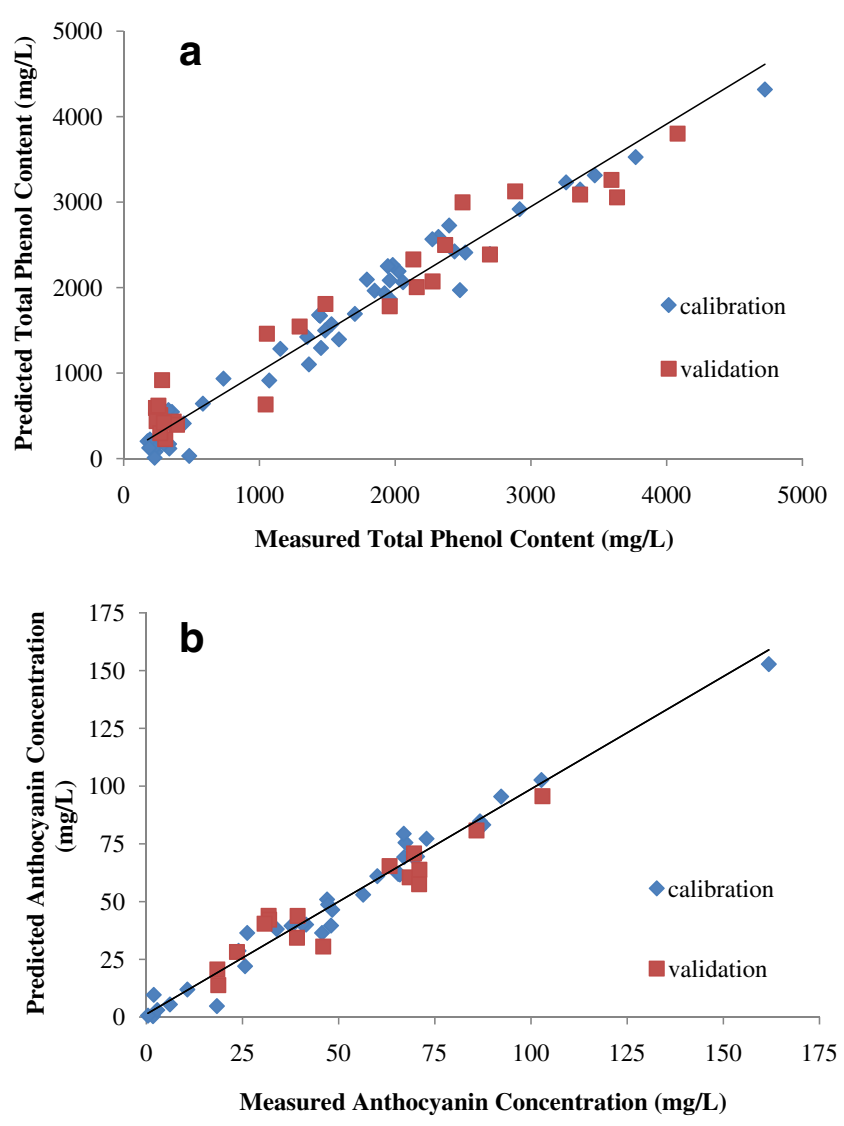

Fig. 2 PLS regression curve for actual vs. predicted a total phenol content and $\mathbf{b}$ anthocyanin content of wine samples

2007). As the reference method HPLC was utilised to determine anthocyanins in different forms as opposed to total anthocyanin amount measured in our study. $R^{2}$ values and relative standard deviation (RSD) were calculated for evaluation of the models and it was recorded that RSD values below $6.6 \%$ indicated good repeatability. Except cyanidin3 -glucoside, prediction of anthocyanins from FT-IR spectra and PLS analysis was successful (Soriano et al. 2007).

The model created for brix prediction is composed of two PCs explaining $89.8 \%$ of total variation $(Y)$. The predictive ability of the model constructed for brix values with FT-IR spectral data was not as good as phenol model $\left(R^{2}=0.9\right)$. Even though the slope of the calibration model was 0.9 which is within the range of $0.9-1.1$, the model for prediction of brix provided good prediction with regression coefficient 0.9 and RPD value of 2.69 (Tables 1 and 2).

Titratable acidity and $\mathrm{pH}$ analytical results were also tried to be predicted from mid-IR spectra. As a result of PLS analysis, slope of titratable acidity model was calculated as 0.89. Although correlation coefficient of 0.89 indicated a good prediction RPD value of 1.67 means insufficient prediction (Tables 1 and 2). However, the analysis resulted in a good correlation for $\mathrm{pH}$ prediction with the slope of 0.92 and $R^{2}$ value of 0.92 . The model contains five PCs and showed a good predictive ability with high RPD value (2.8) for $\mathrm{pH}$ values (Table 2). In a study aimed at measuring several enological parameters of wines with near-IR, mid-IR and combination of near and mid-IR data resulted in a prediction model for total acidity with $R^{2}$ values between 0.795 and 0.814 (Urbano Cuadrado et al. 2005).

The colour parameters were also analysed with PLS regression and the best model was obtained for colour intensity. The model of colour intensity was tested with calibration and validation sets and slope of 0.93 and $R^{2}$ value of 0.93 mean excellent predictions for calibration set. However, RPD value of 2.77 indicated good predictability of the model (Tables 1 and 2). The model created for colour parameters prediction contains three PCs explaining $81 \%$ of total variation $(Y)$ and the correlation coefficients for other colour parameters varied between 0.71 and 0.85 range (Table 2). The model created for determination of blue $\%$ provided approximate prediction with regression coefficient of 0.73 and RPD value of 2.22 (Tables 1 and 2). The predictive ability of the models for prediction of tint, red $\%$ and yellow $\%$ were insufficient with RPD values below 2.0 (Table 2). In a study performed with 145 commercial red wines relations between FT-IR spectra and total wine colour, polymeric pigments, total anthocyanins and co-pigmentation index were established with PLS analysis, as a result, total colour, anthocyanin and polymeric pigment prediction models have $R^{2} \geq 0.82$. However, copigmentation index was not very well predicted with $R^{2}=0.57$ (Laghi et al. 2011).

A previous study, which was performed as an application of mid-IR spectrometry in wine analysis, 327 German wines from vintages of 1989 to 2001 were analysed with reference methods and also scanned with FT-IR to obtain spectral data (Patz et al. 2004). A large group of wine parameters like total phenol, relative density, glycerol, total acidity, $\mathrm{pH}$, alcohol\%, conductivity, sugars (fructose, glucose) and antioxidant activity were successfully predicted from FT-IR spectral data in combination with multivariate data analysis (Patz et al. 2004). In another study, near-IR spectroscopy and multivariate analysis was studied for determination of 15 enological parameters (Urbano-Cuadrado et al. 2004). A total of 180 red, rose and white wines were analysed with analytical methods as reference methods and near-IR spectrometry was used to create models for determination of the parameters in combination with PLS regression analysis. Accurate predictions were observed for determination of ethanol, volumic mass, total acidity, $\mathrm{pH}$, glycerol, colour, tonality (tint) and total polyphenol index by the created equations with high $R^{2}$ values and SECV values close to the reference methods (Urbano-Cuadrado et al. 2004).

As a result of the FT-IR application combined with multivariate data analysis for prediction of the several basic enological parameters, a high performance was achieved 
for some of the selected chemical parameters of wines. With this rapid method, a group of wine quality parameters were determined within a short time simultaneously. Thus, this method can be an alternative to determine some important enological parameters.

\section{Prediction of Raki Quality Parameters}

Statistical analysis of all the measured parameters including $\mathrm{pH}$, brix, electrical conductivity, total phenol content and sugar content were performed separately as it was done for wine samples. Accuracy of the PLS models were again assessed using $R^{2}$, RPD and slope of the equations determined for the calibration sets (Table 3). PLS analysis was applied to establish a relation between FT-IR data as $X$ variables and chemical parameters obtained by analytical methods as $Y$ variables.

$\mathrm{pH}$ value prediction model from FT-IR data constructed with PLS regression was explained with three PCs. The regression coefficient of the model was found as 0.99 for calibration set and 0.92 for validation set. RPD value (2.73) indicated a good prediction. Since the slope of the regression line was 0.99 the model is considered as very reliable according to Table 1 (Fig. 3a).

PLS analysis results for prediction of brix value are shown in Table 3. Highly good regression correlation $\left(R^{2}=0.99\right)$ was obtained using calibration set, and also regression coefficient of validation set was 0.98 . Two PCs were enough for the prediction model. Slope of the brix prediction model was 0.99 and RPD value was calculated as 6.46. Therefore, according to all criteria brix model could be considered to provide excellent prediction. Prediction of parameters such as total soluble solids, $\mathrm{pH}$ and total phenolics for white grape juice from FT-IR spectra using PLS also resulted in successful correlations (Shah et al. 2010).

Prediction of electrical conductivity from FT-IR spectral data with PLS analysis was carried out with three PCs. Correlation coefficients for calibration and validation sets are 0.98 and 0.76 , respectively. RPD value was obtained as 2 . Although the slope of the electrical conductivity prediction model was 0.99 , the other results indicate a model with sufficient prediction. The values of electrical conductivity have big differences between each other, thus small value of RMSEC compared with the value of RMSEP could indicate


Fig. 3 PLS regression curve for actual vs. predicted $\mathbf{a} \mathrm{pH}$ and $\mathbf{b}$ sugar content of raki samples

an over-fitting of PLS model. Actually, electrical conductivities of a raki and an ouzo having mastic are quite large compared to other samples. Therefore, a model was also fitted by eliminating these two data points. As a result, a model with $R^{2}$ values of calibration and validation of 0.98 and 0.24 , respectively, was obtained. RPD value of this model was 0.96 and slope was 0.95 . Also, RMSEC and RMSEP values were calculated as 0.99 and 3.95, respectively. Therefore, FT-IR data did not provide a good prediction for electrical conductivity.

The model created for total phenol prediction consisted of three PCs. Regression coefficients for calibration and validation sets were 0.99 and 0.97 , respectively. Slope of 0.99 and RPD value of 5.42 indicated a good predictive
Table 3 Summary of statistical results for PLS analysis of raki samples

\begin{tabular}{lccccccc}
\hline Parameter & Number of PCs & $R^{2}(\mathrm{cal})$ & $R^{2}(\mathrm{val})$ & Slope & RMSEC & RMSEP & RPD \\
\hline $\mathrm{pH}$ & 3 & 0.99 & 0.92 & 0.99 & 0.07 & 0.22 & 2.73 \\
Brix (\%) & 2 & 0.99 & 0.98 & 0.99 & 0.07 & 0.10 & 6.46 \\
Electrical conductivity $(\mu \mathrm{S} / \mathrm{cm})$ & 3 & 0.99 & 0.76 & 0.99 & 3.12 & 8.31 & 2.01 \\
Total phenol $(\mathrm{mg} / \mathrm{L})$ & 3 & 0.99 & 0.97 & 0.99 & 1.96 & 4.49 & 5.42 \\
Sugar $(\mathrm{ppm})$ & 3 & 0.99 & 0.98 & 0.99 & 523.60 & 464.70 & 7.06 \\
\hline
\end{tabular}


model for total phenol content according to criteria given in Table 1. RMSEC and RMSEP values were not very large.

Excellent prediction of sugar content was obtained as a result of correlating FT-IR data with values obtained analytically (Fig. 3b). The first criterion of slope for prediction model is 0.99 which is between 0.9 and 1.1 and indicates a very reliable model. Secondly, validation and prediction set regression coefficients indicated a highly reliable model because the results are not only higher than 0.9 but also close to each other. Lastly, according to RPD value of 7.05 which is higher than 3, model has very good prediction. Since two ouzo samples have much higher sugar content compared to raki samples a model with only raki samples was also constructed. Values for calibration $R^{2}$ of 0.98 , validation $\mathrm{R}^{2}$ of 0.99 , slope of 0.98 , RMSEC of 228 and RMSEP of 200 showed that this model also has excellent prediction ability according to criteria set in Table 1 . In a study performed with Chinese rice wine, sugar and acid composition were predicted using mid-IR spectroscopy in combination with PLS regression and the models developed have regression coefficients of 0.821-0.991 (Shen et al. 2011).

There are several studies in literature that aim to predict various quality parameters of alcoholic beverages with IR data using chemometric techniques. PLS regression methods were applied for calibration based on full infrared spectra and selected efficient wavenumbers for red wine samples (Pizarro et al. 2011). The high quality of the calibration models were obtained for both total acidity (22 significant wavenumbers) and volatile acidity (11 selected predictor variables). Furthermore, FT-IR spectra and PLS method were proposed as the quality control and authenticity assessment tools for spirit drinks and beer (Lachenmier 2007). Strong correlation with reference methods and great accuracy were found for spirit parameters like density, ethanol, methanol, ethyl acetate, propanol-1, isobutanol and 2-/3methyl-1-butanol contents $\left(R^{2}=0.90-0.98\right)$, for beer parameters of ethanol content, density, original gravity and lactic acid content $\left(R^{2}=0.97-0.98\right)$. On the other hand, beer parameters such as $\mathrm{pH}$, bitterness unit and EBC colour $\left(R^{2}=0.63-0.75\right)$ showed lower correlation and accuracy.

PLS regression technique using FT-IR spectral data provided successful results for prediction of several chemical parameters of raki. As a result, a relationship between FT-IR spectra and some chemical analyses was obtained; therefore, desired chemical parameters could be determined in a short time using FT-IR as an alternative method.

\section{Conclusion}

Application of FT-IR spectroscopy in combination with chemometric techniques for the determination of some quality parameters of a fermented (wine) and a distilled (raki) alcoholic beverage provided promising results. Use of this rapid technique in quality control laboratories could be beneficial for quality evaluation of these types of alcoholic beverages.

\section{References}

Anli RE, Bayram M (2010) Food Rev Int 26:246

Bauer R, Nieuwoudt H, Bauer FF, Kossmann J, Koch KR, Esbensen KH (2008) Anal Chem 80:1371

Bevin CJ, Fergusson AJ, Perry WB, Janik LJ, Cozzolino D (2006) J Agric Food Chem 54:9713

Castellari M, Versari A, Spinabelli U, Galassi S, Amati A (2000) J Liq Chromatogr RT 23:2047

Cozzolino D, Kwiatkowski MJ, Parker M, Cynkar WU, Dambergs RG, Gishen M, Herderich MJ (2004) Anal Chim Acta 513:73

Eriksson L, Erik J, Kettaneh-Wold N (2000) Multivariate data analysis. Principals and applications. Umetrics AB, Sweden

Esbensen KH, Guyot D, Westad F, Houmoller LP (2002) Multivariate data analysis: in practice: an introduction to multivariate data analysis and experimental design. Camp Process AS, Oslo

Fernández K, Agosin E (2007) J Agric Food Chem 55:7294

Ismail AA, van de Voort FR, Sedman J (1997) Fourier transform infrared spectroscopy: principles and applications. In Techniques and instrumentation in analytical chemistry, volume 18, Elsevier, Amsterdam

Kelebek H, Canbas A, Cabaroglu T, Selli S (2007) Food Chem 105:334

Lachenmier DW (2007) Food Chem 101:825

Laghi L, Versari A, Parpinello G, Nakaji DY, Boulton R (2011) Food Anal Methods 4:619

Lee VS, Tue-ngeun P, Traisaith P, Prasitwattanaseree S, Nimmanpipug P, Chaijaruwanich J, Maejo (2009) Int J Sci Technol 3:446

Moreira JL, Santos L (2004) Anal Chim Acta 513:263

Nieuwoudt HH, Pretorius IS, Bauer FF, Nel DG, Prior BA (2006) J Microbiol Meth 67:248

Palma M, Barroso CG (2002) Talanta 58:265

Patz CD, Blieke A, Ristow R, Dietrich H (2004) Anal Chim Acta 513:81

Pizarro C, González-Sáiz JM, Esteban-Díez I, Orio P (2011) Anal Bioanal Chem 399:2061

Pontes MJC, Santos SRB, Araujo MCU, Almeida LF, Lima RAC, Gaiao EN, Souto UTCP (2006) Food Res Int 39:182

Saeys W, Mouazen AM, Ramon H (2005) Biosyst Eng 91:393

Shah N, Cynkar W, Smith P, Cozzolino D (2010) J Agric Food Chem 58:3279

Shen F, Ying Y, Li B, Zheng Y, Hu J (2011) Food Res Int 14:1521

Slinkard K, Singleton VL (1977) Am J Enol Viticult 28:49

Soriano A, Pérez-Juan PM, Vicario A, González JM, Pérez-Coello MS (2007) Food Chem 104:1295

Tamaki Y, Mazza G (2011) J Agric Food Chem 59:504

Urbano Cuadrado M, Luque de Castro MD, Perez Juan PM, GomezNieto MA (2005) Talanta 66:218

Urbano-Cuadrado M, Luque de Castro MD, Pérez-Juan PM, GarcíaOlmo J, Gómez-Nieto MA (2004) Anal Chim Acta 527:81

Versari GP, Parpinello, Scazzina F, Del Rio D (2010) Food Control 21:786

Zornoza R, Guerrero C, Mataix-Solera J, Scow KM, Arcenegui V, Mataix Beneyto J (2008) Soil Biol Biochem 40:1923 